\title{
The First Total Synthesis of Artemisinin (Qinghaosu)
}<smiles>C=C(C)[C@H]1CC[C@@H](C)C[C@H]1O</smiles>

(-)-isopulegol<smiles>CC1CCC(C(C)CO)C(=O)C1</smiles>

1. LDA, THF, $0^{\circ} \mathrm{C}$ then $\mathrm{B}, 67 \%$ yield

2. C (10 equiv) $\mathrm{dr}=8: 1,89 \%$ yield $\mathrm{CH}_{2} \mathrm{Cl}_{2}$, r.t., $h v, 60 \%$ yield

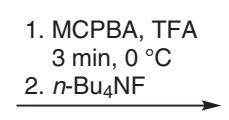
$68 \%$ over 2 steps Peterson olefination
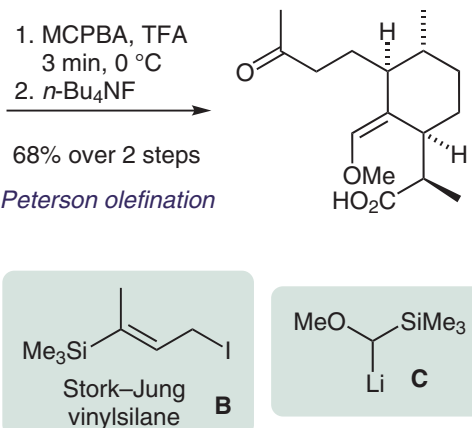<smiles>COC(Cl)C(C)C</smiles>
C

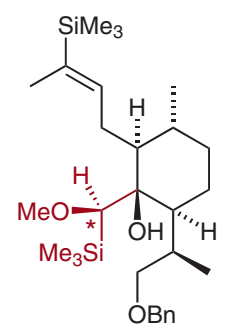

$\mathrm{O}_{2}$, methylene blue,
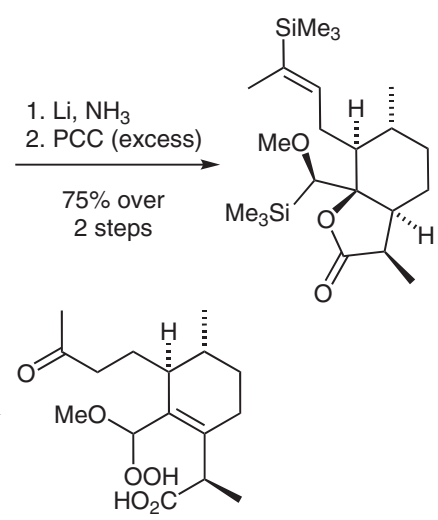

$\mathrm{O}_{2}$, methylene blue $\mathrm{MeOH},-78^{\circ} \mathrm{C}, h v$

then $\mathrm{HCO}_{2} \mathrm{H}, \mathrm{CH}_{2} \mathrm{Cl}_{2}$ $0{ }^{\circ} \mathrm{C}, 24 \mathrm{~h}, 30 \%$ yield

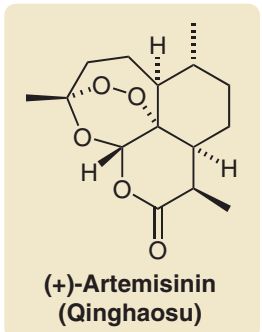

Category

Chemistry in

Medicine and Biology

\section{Key words}

antimalarial

photooxygenation

kinetic resolution
Significance: In a great collaborative effort, 'project 523' aimed to seek new treatments for malaria under the direction of Youyou Tu in the 1970s. Using herbal extracts from traditional Chinese medicine as a lead, sweet wormwood (Artemisia annua L.) was found to be effective against malaria. Isolation and characterization of artemisinin (qinghaosu) were achieved by 1973 . The first syntheses of this complex 1,2,4-trioxane containing natural product were reported by W. Hofheinz and G. Schmid and independently by W.-S. Zhou and co-workers in 1983 (Xu et al. Acta Chim. Sin. 1983, 41, 574; Xu et al. Acta Chim. Sin. Engl. Ed. 1983, 1, 98). Both syntheses confirmed the previously elucidated structure. Nowadays, artemisinin derivatives remain the most effective antimalarial medication and, to continuously secure its supply, various modern synthesis technologies are being investigated.
Comment: Intermediate A was synthesized in five steps from (-)-isopulegol involving hydroboration, oxidation, and protecting-group manipulation. Alkylation with the Stork-Jung vinylsilane (B) and addition of $\mathbf{C}$ were achieved with high diastereoselectivity under substrate control. However, 10 equivalents of $\mathbf{C}$ were required to increase the diastereomeric ratio through kinetic resolution of the racemic organolithium reagent by the chiral ketone. Debenzylation followed by oxidation of the alcohol and intermediary lactol afforded the lactone. Vinylsilane oxidation followed by a stereoselective Peterson olefination afforded the penultimate intermediate. Based on reports by Asveld and Kellogg (J. Am. Chem. Soc. 1980, 102, 3644), the authors examined the photooxygenation at low temperature in methanol, followed by treatment with formic acid. This enabled the direct formation of $(+)$-artemisinin. 